\title{
ON THE DETERMINATION OF CHARACTERISTIC VALUES FOR A CLASS OF STURM-LIOUVILLE PROBLEMS
}

\author{
BY \\ RIChaRd BeLlMaN
}

\section{Introduction}

In this paper we are interested in the problem of determining the characteristic values of the Sturm-Liouville equation

$$
u^{\prime \prime}+\lambda a(t) u=0, \quad u(0)=u(1)=0 .
$$

It will be clear from what follows that the methods we discuss can be applied to questions of this type involving quite general boundary conditions, as long as the interval is finite.

There are, at present, a number of powerful techniques available for treating problems of this genre, based upon variational techniques, and upon matrix techniques applied to a finite difference version of the foregoing differential equation.

The variational approach depends upon the fact that if $a(t)$ satisfies a reasonable condition such as

$$
0<a^{2} \leqq a(t) \leqq b^{2}<\infty, \quad 0 \leqq t \leqq 1,
$$

then the characteristic values, $\lambda_{1}<\lambda_{2}<\cdots$, are the respective relative minima of the functional

$$
J(u)=\int_{0}^{1} u^{\prime 2} d t / \int_{0}^{1} a(t) u^{2} d t
$$

as $u$ ranges over the space of functions for which the integrals exist and for which $u(0)=u(1)=0$.

In particular,

$$
\lambda_{1} \leqq \int_{0}^{1} u^{\prime 2} d t / \int_{0}^{1} a(t) u^{2} d t
$$

for all functions $u(t)$ satisfying the prescribed boundary conditions. We thus have a means of obtaining upper bounds for $\lambda_{1}$ which turn out to be remarkably accurate even for simple choices of trial functions $u(t)$.

Another method is based upon using equations of the form

$$
u_{n+2}-2 u_{n+1}+u_{n}+\lambda \Delta^{2} a_{n} u_{n}=0
$$

$u(0)=u(N)=0$, and applying any of a number of methods used to derive the characteristic roots and vectors of a symmetric matrix. For a detailed discussion of these methods, and others, we refer to the book by Collatz $[2]$.

Received July 5, 1957; received in revised form September 18, 1957. 
There is, however, a significant difference between a problem of this type, and the Sturm-Liouville problem described above. This is due to the fact that it is quite easy to find asymptotic solutions to (1) for large $\lambda$, and thus, approximate expressions for the higher characteristic values.

Let, for simplicity of notation, $a(t)=q^{2}(t)$; then the Liouville transformation (cf. [1], p. 109) $s=\int_{0}^{t} q\left(t_{1}\right) d t_{1}$, converts

$$
u^{\prime \prime}+\lambda q^{2}(t) u=0
$$

into

$$
\frac{d^{2} u}{d s^{2}}+\frac{q^{\prime}(t)}{q^{2}(t)} \frac{d u}{d s}+\lambda u=0 .
$$

The further transformation

converts (7) into

$$
v=u \sqrt{q(t)}=u \exp \left\{\frac{1}{2} \int \frac{q^{\prime}(t)}{q^{2}(t)} d s\right\}
$$

$$
\frac{d^{2} v}{d s^{2}}+\left[\lambda-\frac{1}{2} \frac{d}{d s}\left(\frac{a^{\prime}(t)}{a^{2}(t)}\right)-\frac{1}{4}\left(\frac{a^{\prime}(t)}{a^{2}(t)}\right)^{2}\right] v=0 .
$$

The new boundary conditions are

$$
v(0)=0, \quad v\left(\int_{0}^{1} q(t) d t\right)=0 .
$$

Writing (9) in the form

$$
v^{\prime \prime}(s)+(\lambda+b(s)) v(s)=0,
$$

we know that we can find asymptotic developments for $v(s)$ starting from the integral equation

$$
v(s)=c_{1} \cos \lambda^{1 / 2} s+c_{2} \sin \lambda^{1 / 2} s-\int_{0}^{s} \frac{\left[\sin \lambda^{1 / 2}(s-r)\right]}{\lambda^{1 / 2}} b(r) v(r) d r
$$

and iterating (cf. [1], pp. 55-62 for analogous treatment over the infinite interval). Approximate values of $\lambda$ are now determined by means of the constraint $v\left(\int_{0}^{1} q(t) d t\right)=0$. Thus, the higher characteristic values have the principal term

$$
\lambda_{n} \cong n^{2} \pi^{2} /\left(\int_{0}^{1} q(t) d t\right)^{2}
$$

To obtain more precise results, we can use further terms of the asymptotic series derived from (12), and we can combine this with numerical integration of (1).

It follows from these considerations that the greatest difficulty is experienced in obtaining accurate estimations of the first characteristic value. In many investigations this is all that is desired.

We wish to present a new method, suitable for hand or digital computer calculation, which furnishes monotone convergence, through sequences of 
upper and lower bounds, to the smallest characteristic value. Similar sequences can be used to obtain monotone convergence to products of the form $\prod_{i=1}^{k} \lambda_{i}$. The method has the advantage of permitting $\lambda_{1}$ to be determined to a high degree of accuracy.

To illustrate these techniques, we consider the equation

$$
u^{\prime \prime}+\lambda(1+t) u=0, \quad u(0)=u(1)=0,
$$

which is connected with Airy's function, or Bessel functions of order $\frac{1}{3}$. The computations were performed with the assistance of Marvin Shapiro and Oliver Gross of the Rand Corporation.

\section{The equation determining the characteristic values}

Let us note in passing that the method we use is an application of an approach we have used, in various lecture courses on differential equations, to derive the fundamental results of Sturm-Liouville theory.

Consider the linear differential equation

$$
u^{\prime \prime}+\lambda a(t) u=0, \quad u(0)=0, \quad u^{\prime}(0)=1 .
$$

The solution of this initial value problem may be obtained over $0 \leqq t \leqq 1$ as a power series in $\lambda$ in the form

$$
u=t+\sum_{n=1}^{\infty} u_{n}(t) \lambda^{n},
$$

where the sequence of coefficient functions $\left\{u_{n}(t)\right\}, n=1,2, \cdots$, may be determined by means of the recurrence relations

$$
\begin{aligned}
& u_{0}(t)=t, \\
& u_{n}(t)=-\int_{0}^{t}(t-s) u_{n-1}(s) a(s) d s, \quad n=1,2, \cdots .
\end{aligned}
$$

It is easy to see that $u$, as defined by (2), is an analytic function of $\lambda$ for all finite $\lambda$ for $0 \leqq t \leqq 1$. The roots of the equation

$$
f(\lambda)=u(1)=1+\sum_{n=1}^{\infty} u_{n}(1) \lambda^{n}=0
$$

are the desired characteristic values.

\section{Discussion}

If we assume that the sequence of coefficients is determined by means of either a hand or machine computation, a matter we will discuss again below, there is the problem of determining the first few roots of the equation in (2.4).

This is a problem which can be treated in several ways. It would seem that an efficient procedure would be to use the sequences we shall describe presently to obtain reasonably accurate estimates for the characteristic values, and then use Newton's method, or a modification, to obtain very accurate values. 


\section{Analytic preliminaries}

Referring to the equation in (2.1), we easily see that

$$
|u(t)| \leqq e^{k|\lambda|^{1 / 2}},
$$

for $0 \leqq t \leqq 1$, where $k$ is a constant. Consequently, the Weierstrass factorization of $f(\lambda)$ takes the form

$$
f(\lambda)=\prod_{i=1}^{\infty}\left(1-\lambda / \lambda_{i}\right) .
$$

As we know, $\lambda_{n}=O\left(n^{2}\right)$ as $n \rightarrow \infty$, in view of the assumptions we have made concerning $a(t)$ in (1.2).

Our aim is now, by following the technique used by Newton to relate the sums of the powers of the roots and the elementary symmetric functions, which are the coefficients, to obtain relations for the sums

$$
b_{r}=\sum_{i=1}^{\infty} 1 / \lambda_{i}^{r}, \quad r=1,2, \cdots,
$$

in terms of the coefficients $u_{n}(1)$.

It is clear that

$$
\begin{aligned}
\log f(\lambda)=\sum_{i=1}^{\infty} \log \left(1-\lambda / \lambda_{i}\right) & =-\sum_{r=1}^{\infty}\left(\lambda^{r} / r\right)\left\{\sum_{i=1}^{\infty} 1 / \lambda_{i}\right\} \\
& =-\sum_{r=1}^{\infty} \lambda^{r} b_{r} / r,
\end{aligned}
$$

for $|\lambda|<\lambda_{1}$.

It is important then to obtain the coefficients of the expression of $\log f(\lambda)$. Although this can be done directly, it is easier to proceed as follows. Write

$$
\log f(\lambda)=\sum_{k=1}^{\infty} c_{k} \lambda^{k}
$$

Then

$$
f^{\prime}(\lambda) / f(\lambda)=\sum_{k=1}^{\infty} k c_{k} \lambda^{k-1}
$$

whence

$$
\sum_{n=1}^{\infty} n u_{n}(1) \lambda^{n-1}=\left(\sum_{k=1}^{\infty} k c_{k} \lambda^{k-1}\right)\left(1+\sum_{n=1}^{\infty} u_{n}(1) \lambda^{n}\right),
$$

whence we obtain the well-known recurrence relations

$$
n u_{n}=n c_{n}+\sum_{k=1}^{n-1} k c_{k} u_{n-k} .
$$

These permit us to calculate the $c_{n}$ in a very simple fashion once the sequence $\left\{u_{n}(1)\right\}$ has been determined, and thence the $b_{n}$.

\section{Inequalities}

Let us now show that the sequence $\left\{b_{k}\right\}$ can be used to obtain sequences which converge monotonically from above and below to the first characteristic value $\lambda_{1}$.

THeOREM 1. We have the inequalities

$$
b_{k} / b_{k+1}>\lambda_{1}>1 / b_{k}^{1 / k}, \quad k=1,2, \cdots .
$$


The sequence $\left\{b_{k} / b_{k+1}\right\}$ is monotone decreasing; the sequence $\left\{1 / b_{k}^{1 / k}\right\}$ is monotone increasing, and

$$
\lambda_{1}=\lim _{k \rightarrow \infty} b_{k} / b_{k+1}=\lim _{k \rightarrow \infty} 1 / b_{k}^{1 / k} .
$$

Proof. The monotone character of the ratio $b_{k} / b_{k+1}$ follows directly from Schwarz's inequality, since

$$
\begin{aligned}
b_{k}^{2} & =\left(\sum_{i=1}^{\infty} 1 / \lambda_{1}^{k}\right)^{2}=\left(\sum_{i=1}^{\infty} 1 / \lambda_{i}^{(k+1) / 2} \lambda_{i}^{(k-1) / 2}\right)^{2} \\
& \leqq\left(\sum_{i=1}^{\infty} 1 / \lambda_{i}^{k+1}\right)\left(\sum_{i=1}^{\infty} 1 / \lambda_{i}^{k-1}\right)=b_{k+1} b_{k-1} .
\end{aligned}
$$

The monotone behavior of $b_{k}^{1 / k}$ is a consequence of the well-known inequality

$$
\left(\sum_{i=1}^{\infty} x_{i}\right) \geqq\left(\sum_{i=1}^{\infty} x_{i}^{2}\right)^{1 / 2} \geqq\left(\sum_{i=1}^{\infty} x_{i}^{3}\right)^{1 / 3} \geqq \cdots,
$$

for any set of nonnegative $x_{i}$.

The proof of the limiting relation is clear.

\section{Rate of convergence}

Since

(1) $\frac{b_{k}}{b_{k+1}}=\frac{\left(1 / \lambda_{1}^{k}\right)\left[1+\left(\lambda_{1} / \lambda_{2}\right)^{k}+\cdots\right]}{\left(1 / \lambda_{1}^{k+1}\right)\left[1+\left(\lambda_{1} / \lambda_{2}\right)^{k+1}+\cdots\right]}=\lambda_{1}\left[1+\left(\lambda_{1} / \lambda_{2}\right)^{k}-\left(\lambda_{1} / \lambda_{2}\right)^{k+1}+\cdots\right]$, we see that

$$
b_{k} / b_{k+1}-\lambda_{1} \cong \lambda_{1}\left(\lambda_{1} / \lambda_{2}\right)^{k}
$$

for large $k$.

Similarly,

(3) $b_{k}^{1 / k}=\left(1 / \lambda_{1}\right)\left(1+\left(\lambda_{1} / \lambda_{2}\right)^{k}+\cdots\right)^{1 / k} \cong\left(1 / \lambda_{1}\right)\left(1+k\left(\lambda_{1} / \lambda_{2}\right)^{k}\right)$

for large $k$.

It is to be expected that $b_{k} / b_{k+1}$ will furnish a better approximation to $\lambda_{1}$ for large $k$.

\section{Discussion}

For the case where $a(t) \equiv 1, \lambda_{1} / \lambda_{2}=\frac{1}{4}$. Consequently, in general, the rate of convergence of these sequences will not be too rapid. There are two things we can do to obtain more accurate estimations of $\lambda_{1}$. In the first place, we can use the root-squaring technique. Since

$$
f(\lambda)=\prod_{i=1}^{\infty}\left(1-\lambda / \lambda_{i}\right)
$$

we see that

$$
f_{1}(\lambda)=f\left(\lambda^{1 / 2}\right) f\left(-\lambda^{1 / 2}\right)=\prod_{i=1}^{\infty}\left(1-\lambda / \lambda_{i}^{2}\right) .
$$

Using the power series development for $f_{1}(\lambda)$ we obtain a sequence $\left\{b_{k}^{\prime}\right\}$ with

$$
\lim _{k \rightarrow \infty} b_{k}^{\prime} / b_{k+1}^{\prime}=\lambda_{1}^{2},
$$

and a rate of convergence depending upon $\left(\lambda_{1} / \lambda_{2}\right)^{2}$. 
Alternatively, once we have an estimate for $\lambda_{1}$ with an accuracy of 1 in $10^{-s}$, we can then turn to the power series for $f(\lambda)$ and use the Newton approximation technique,

$$
\lambda_{1}^{(n+1)}=\lambda_{1}^{(n)}-f\left(\lambda_{1}^{(n)}\right) / f^{\prime}\left(\lambda_{1}^{(n)}\right) .
$$

This will yield a further approximation with accuracy of essentially 1 in $10^{-2 s}$. Continued use of this technique is limited only by the number of $u_{n}(1)$ which are computed, and the accuracy of this computation. There is no difficulty involved in using this technique here, since we know from theoretical considerations that the roots of $f(\lambda)$ are simple.

\section{Inequalities for $\prod_{i=1}^{R+1} \lambda_{i}$}

Similar upper bounds can be obtained for the products $\prod_{i=1}^{R+1} \lambda_{i}$, $R=1,2, \cdots$.

Consider the determinant

$$
b_{k}^{(R)}=\left|\begin{array}{cccc}
b_{k} & b_{k+1} & \cdots & b_{k+R} \\
\vdots & & & \\
b_{k+R} & b_{k+R+1} & \cdots & b_{k+2 R}
\end{array}\right|, \quad R=1,2, \cdots .
$$

It is not difficult to show that

$$
\lim _{k \rightarrow \infty} b_{k}^{(R)} / b_{k+1}^{(R)}=\lim _{k \rightarrow \infty}\left(b_{k}^{(R)}\right)^{-1 / k}=\lambda_{1} \lambda_{2} \cdots \lambda_{R+1} .
$$

To show that

$$
b_{k}^{(R)} / b_{k+1}^{(R)}>b_{k+1}^{(R)} / b_{k+2}^{(R)},
$$$$
k=1,2, \cdots,
$$

for $R=1,2, \cdots$, we use the well-known fact that the matrix

$$
B_{k}^{(R)}=\left(\begin{array}{cccc}
b_{k} & b_{k+1} & \cdots & b_{k+R} \\
\vdots & & & \\
b_{k+R} & b_{k+R+1} & \cdots & b_{k+2 R}
\end{array}\right)
$$

is positive definite for all $k$ and $R$, and hence that $\left(B_{k}^{(R)}\right)^{-1}$ is positive definite.

The sequence $\left(b_{k}^{(R)}\right)^{-1 / k}$ does not seem to have any simple monotonicity properties.

$$
\text { 9. The equation } u^{\prime \prime}+\lambda(1+t) u=0
$$

Let us now illustrate some of the ideas discussed above by means of the equation

$$
u^{\prime \prime}+\lambda(1+t) u=0, \quad u(0)=u(1)=0 .
$$

The first problem we face is that of computing the sequence $\left\{u_{n}(t)\right\}$ by means of the recurrence relations of (2.3). Since $u(t)$ is an entire function of $\lambda$ for $0 \leqq t \leqq 1$, the coefficients, $u_{n}(t)$, become quite small as $n$ increases. If $a(t) \equiv 1$, the coefficient of $\lambda^{n}$ is $(-1)^{n} /(2 n+1)$ ! Hence, if we are using a digital computer, even one with floating point arithmetic, it is necessary to renormalize. A very simple renormalization is one which sets

$$
v_{n}(t)=(-1)^{n}(2 n+1) ! u_{n}(t) .
$$


Then

$$
\begin{aligned}
& v_{n}(t)=\frac{1}{(2 n+1) 2 n} \int_{0}^{t}(t-s) v_{n-1}(s) a(s) d s, \quad n=1,2, \cdots \\
& v_{0}(t)=1 .
\end{aligned}
$$

Since (3) is equivalent to the differential recurrence relation

$$
v_{n}^{\prime \prime}(t)=a(t) v_{n-1}(t) / 2 n(2 n+1) \quad v_{n}(0)=v_{n}^{\prime}(0)=0,
$$

we can use a Runge-Kutta integration procedure to obtain fairly accurate values of $v_{n}(1)$ (see Table 1 ).

The decision as to how many elements of the sequence $\left\{u_{n}(1)\right\}$ to compute depends upon an a priori estimate of the magnitude of $\lambda_{1}$, the time involved in the computation, the accuracy of the computation, and the accuracy with which $\lambda_{1}$ is desired.

Since $1+t \geqq 1$, we see that $\lambda_{1}<\pi^{2}<10$. Hence the order of magnitude of the last term computed in the power series would be

$$
u_{n}(1) \lambda_{1}^{n}<\frac{53.5}{(21) !} 10^{10}<\frac{10^{2} \cdot 10^{10}}{(20) !}<\frac{10^{2} \cdot 10^{10}}{2^{20} \cdot 10^{20}}=\frac{10^{-8}}{2^{20}}
$$

(using Sterling's approximation). This is more than sufficient, considering

\begin{tabular}{|c|c|c|c|c|}
\hline$k$ & $b_{k}$ & \multicolumn{2}{|c|}{$b_{k} / b_{k+1}$} & $\begin{array}{c}b_{k}^{-1 / k} \\
\text { (slide rule evaluation) }\end{array}$ \\
\hline 1 & 25.0000 & 9.921 & 26 & 4.00 \\
\hline 2 & 251.984 & 6.958 & 90 & 6.30 \\
\hline 3 & 3621.03 & 6.632 & 47 & 6.51 \\
\hline 4 & 54595.5 & 6.567 & 79 & 6.54 \\
\hline 5 & 831261.0 & 6.553 & 06 & 6.55 \\
\hline 6 & 12685100.0 & 6.549 & 54 & - \\
\hline 7 & $193679 \times 10^{3}$ & 6.548 & 66 & - \\
\hline 8 & $29575 \times 10^{5}$ & & & 一 \\
\hline
\end{tabular}
TABLE 1

\begin{tabular}{r|rrrr}
\hline$n$ & \multicolumn{4}{|c}{$v_{n}(1)=(-1)^{n}(2 n+1) ! u_{n}(1)$} \\
\hline 0 & 1. & 000 & 000 & 000 \\
1 & 1. & 499 & 999 & 92 \\
2 & 2. & 238 & 094 & 66 \\
3 & 3. & 333 & 330 & 15 \\
4 & 4. & 960 & 358 & 93 \\
5 & 7. & 378 & 146 & 87 \\
6 & 10. & 971 & 261 & 4 \\
7 & 16. & 310 & 824 & 0 \\
8 & 24. & 244 & 529 & 3 \\
9 & 36. & 028 & 967 & 6 \\
10 & 53. & 522 & 379 & 4 \\
\hline
\end{tabular}

TABLE 2 
TABLE 3

\begin{tabular}{c|c|c|c}
\hline$k$ & $b_{k}^{(1)}=b_{k} b_{k+2}-b_{k+1}^{2}$ & $b_{k}^{(1)} / b_{k+1}^{(1)}$ & $\left(b_{k}^{(1)}\right)^{-1 / k}$ \\
\cline { 2 - 4 } 1 & 27030.0 & 418.85 & .37 \\
2 & 645330.0 & 219.85 & 12.45 \\
3 & $29353 \times 10^{3}$ & 188.93 & 32.45 \\
4 & $15537 \times 10^{5}$ & 179.34 & 89.5 \\
5 & $86634 \times 10^{6}$ & - & - \\
\hline
\end{tabular}

the inaccuracy involved in numerical integration, for the determination of $\lambda_{1}$, and is sufficient for the determination of $\lambda_{2} \leqq 4 \pi^{2}$.

The next step is to compute the sequence of coefficients in $\log f(\lambda)$, namely $\left\{b_{k}\right\}$, using (4.8). The results are given in Table 2, together with the ratios $b_{k} / b_{k+1}$ and the roots $b_{k}^{-1 / k}$.

For the purposes of using the Newtonian scheme mentioned above, (7.4), we see that $b_{4} / b_{5}$ and $b_{4}{ }^{-1 / 4}$ yield sufficiently good initial approximations with an error of about 1 in 600 . One or two applications of (7.4) would yield $\lambda_{1}$ to an accuracy sufficient for most purposes.

The convergence of the sequences for $\lambda_{1} \lambda_{2}$ is much less rapid, as is to be expected. The results are shown in Table 3.

Using the value of $\lambda_{1}$ obtained above, we obtain a first approximation of $\lambda_{2} \cong 27$. From the monotonicity of the ratios, we know that $\lambda_{2}$ is actually less than this. An application of Newton's approximation will yield a greatly improved result.

Note that $\lambda_{2}$ is sufficiently large so that the asymptotic techniques discussed in $\$ 1$ can be used to provide an independent check of the accuracy of the first approximation to $\lambda_{2}$.

\section{Alternate computational scheme for polynomial coefficients}

In what has preceded, we have spoken in terms of numerical evaluation of the sequence $\left\{u_{n}(t)\right\}$. Although this procedure has the great advantage of straightforwardness and simplicity, via hand computation or digital computation, it suffers from the fact that errors of integration arise, and grow with each new member of the sequence.

Consequently, it is worth noting a special, but important, case in which we can avoid mechanical quadrature and carry out the entire operation by hand.

Suppose that $a(t)$ is a polynomial of the form

$$
a(t)=a_{0}+a_{1} t+\cdots+a_{k} t^{k} .
$$

It will be clear then that the elements of the sequence $\left\{u_{n}(t)\right\}$ will also be polynomials. Furthermore, it is clear that $u_{n}(t)$ will have the form

$$
u_{n}(t)=a_{0} t^{2 n+1} /(2 n+1) !+\cdots+a_{k n} t^{2 n+k}+\cdots .
$$


Using the recurrence relation of (2.3), we can then obtain linear recurrence relations for the sequence $\left\{a_{k n}\right\}, k=1,2, \cdots ; n=1,2, \cdots$.

There are a number of renormalization questions concerned with the effective calculation of the sequence, and asymptotic relations which can be used to speed the computation. A discussion of these would take us too far afield.

\section{Extension to higher order equations}

Let us now consider the equation

$$
u^{(4)}+\lambda a(t) u=0
$$

with the boundary conditions

$$
u(0)=u^{\prime}(0)=0, \quad u(1)=u^{\prime}(1)=0 .
$$

Proceeding as above, we consider the solution, $u(t, \lambda)$, of the initial value problem

$$
u(0)=0, \quad u^{\prime}(0)=0, \quad u^{\prime \prime}(0)=c_{1}, \quad u^{\prime \prime \prime}(0)=c_{2},
$$

which we can write in the form

$$
u=c_{1} u_{1}(t, \lambda)+c_{2} u_{2}(t, \lambda)
$$

where $u_{1}$ and $u_{2}$ are determined by the initial conditions

$$
\begin{aligned}
& u_{1}(0)=0, \quad u_{2}(0)=0, \quad u_{1}^{\prime}(0)=0, \quad u_{2}^{\prime}(0)=0, \\
& u_{1}^{\prime \prime}(0)=1, \quad u_{2}^{\prime \prime}(0)=0, \quad u_{3}^{\prime \prime \prime}(0)=0, \quad u_{3}^{\prime \prime \prime}(0)=1 \text {. }
\end{aligned}
$$

As before, there is no difficulty in obtaining the power series developments in terms of $\lambda$ for the functions $u_{1}$ and $u_{2}$.

Applying the boundary conditions in (2), we obtain the simultaneous equations

$$
c_{1} u_{1}(1, \lambda)+c_{2} u_{2}(1, \lambda)=0, \quad c_{1} u_{1}^{\prime}(1, \lambda)+c_{2} u_{2}^{\prime}(1, \lambda)=0,
$$

whence the determining equation for $\lambda$ is

$$
f(\lambda)=\left|\begin{array}{ll}
u_{1}(1, \lambda) & u_{2}(1, \lambda) \\
u_{1}^{\prime}(1, \lambda) & u_{2}^{\prime}(1, \lambda)
\end{array}\right|=0 .
$$

From here on, the argumentation is as before.

\section{REFERENCES}

1. R. Bellman, Stability theory of differential equations, New York, McGraw-Hill, 1953.

2. L. Collatz, Numerische Behandlung von Differentialgleichungen, Berlin, Springer, 1951.

The Rand Corporation

Santa Monica, California 